\title{
Leader Orientation, Leader Behavior, Group Effectiveness and Situational Favorability: An Empirical Extension of the Contingency Model ${ }^{1}$
}

\author{
Larry K. Michaelsen ${ }^{2}$ \\ Center for Research on Utilization of Scientific Knowledge, University of Michigan
}

The relationship between work group effectiveness, leaders' behavior, and leaders' interpersonal relations orientation scores in three levels of situational favorability were studied. Subjects were members and supervisors of 119 work groups in the maintenance and production departments of a metal fabricating plant. It was found that (a) in a very unfavorable situation, supervisors directed most of their behavior toward the achievement of their primary goals, while in a very favorable situation, they concentrated less on the achievement of primary goals and more on the achievement of secondary goals; and (b) groups supervised by task-oriented leaders were more effective in situations of either high or low favorability, while groups supervised by interpersonal relations-oriented leaders were more effective in situations of intermediate favorability.

During the last 50 years, the study of leadership behavior and group effectiveness has seen a number of approaches popularized, empirically tested, and then modified or abandoned. Among the most notable of these are what might be called the "great man" theories, characterized by numerous attempts to identify a set of traits that would distinguish successful leaders, and the theories espousing some "great method" such as the human relations approach stemming from the work of Elton Mayo. As empirical evidence accumulated, it became apparent that neither a "great man" nor a "great method" could provide effective leadership in all sets of circumstances. As a result, more recent examinations of leadership behavior and group effectiveness have focused on its interactional and situational character. The primary question of research on leadership has shifted from "what is the best kind of leadership?"

${ }^{1}$ Research support from the Office of Naval Research, Organizational Effectiveness Research Programs, is gratefully acknowledged.

The author wishes to thank Stanley Seashore and David Bowers for their expert assistance.

${ }^{2}$ Requests for reprints should be sent to Larry Michaelsen, Center for Research on Utilization of Scientific Knowledge, Institute for Social Research, University of Michigan, Ann Arbor, MI 48106.

Copyright (C) 1973 by Academic Press, Inc. All rights of reproduction in any form reserved. 
to "what kind of leadership works best in what kind of situation?" The development of the "contingency model" by Fiedler and his colleagues at the University of Illinois is probably the most outstanding example of this trend.

The Fiedler model postulates a contingent relationship between group performance and the degree to which leaders tend to view their least preferred coworker in a favorable or an unfavorable light. This relationship between group effectiveness and leaders' Least Preferred Coworker (LPC) scores is moderated by a situational favorability dimension, defined by Fiedler as the degree to which the leadership situation enables the leader to exert influence over his group (Fiedler, 1967, p. 13). The primary components of situational favorability in the model are the quality of leader-member relations, the degree of structure of the task, and the position power of the leader. In the model, these three dimensions are each conceptualized as dichotomies, and, when combined, result in a three-dimensional, eight-celled cube of situations, each deseribing a group which has a certain amount (high or low) of each of these three attributes. The most favorable situation, then, would be in the octant in which leader-member relations scores are good, the group is working on a structured task, and the leader has legitimate authority to reward and punish facilitative and nonfacilitative task behavior on the part of group members.

The theory postulates that groups whose leaders describe their least preferred coworker in a negative manner (low LPC) perform better in very favorable and very unfavorable situations, and that groups whose leaders describe their least preferred coworker in relatively positive terms (high LPC) perform better in conditions of intermediate favorability. This theoretical model, first presented in 1964, has been generally substantiated in a large number of subsequent studies (Fiedler, 1971 (b) ; see also a critique by Graen et al., 1970). In spite of the consistent relationships between LPC and group performance, one of the problems with the model has been an inability to define what LPC is measuring. Fiedler himself states:

It has been extremely difficult to develop an adequate and readily supportable interpretation of ... LPC scores. These scores do not measure attributes which correlate with the usual personality and ability tests or with attitude scales. Nor is there a one-to-one relationship between these scales and behaviors [Fiedler, 1967, p. 45].

Subsequently, a number of studies by Fiedler and his associates have been directed toward the determination of the nature of the relationship between LPC scores and leader behavior.

In 1968, Fiedler stated: 
It has become clear that we are dealing with a motivational measure that manifests itself in different behaviors ... the individual who rates his least preferred co-worker in relatively favorable terms tends to be considerate of the feelings and opinions of his co-workers. . . The low LPC leader ... tends to be task-oriented and structuring in his behavior and concerned with productivity rather than with interpersonal relations [p. 363].

In a later work, Fiedler (1970) concluded that, "This interpretation, based on weak observational data and leader descriptions, turns out to present an approximate but grossly oversimplified picture" [pp. 2-3]. He then offered a somewhat more complex interpretation of the relationship between LPC scores and leader behavior. This interpretation was based on four explicitly stated assumptions:

1. Individuals have a variety of goals of varying importance.

2. Different types of individuals have different goal structures; hence the goals which have primary value for some may have secondary or even negligible importance for others.

3. An individual will attempt to achieve as many of his goals as possible. In situations in which his control and influence are relatively weak or threatened, the individual will tend to concentrate his efforts in securing his primary goals to the neglect of his secondary goals. In situations in which his control and influence are relatively great, he will begin to channel some of his energies toward the attainment of his secondary goals.

4. LPC scores effectively identify two types of individuals, at least with regard to their behavior in task situations. On one end of a continuum are those people who are primarily motivated to seek interpersonal interactions (high LPC), while on the other end are those who are primarily motivated by explicit competition for material and tangible rewards in the work situation (low LPC) (Fiedler, 1970, pp. 4-5).

This interpretation suggests that a leader's LPC score is a measure of one of his "lower order needs," if, indeed, an analogy to Maslow's classical model is appropriate, that "once satisfied is no longer a motivator" (1943). The switch from one form of behavior to another as a situation becomes more difficult is also reminiscent of Blake and Mouton's discussion of dominant and backup leadership styles. They stated:

Observe an individual's behavior in a variety of situations. It becomes clear that even the notion of one dominant style, a single set of managerial assumptions, is not sufficient to eatch the full implication of a person's managerial approach. In addition to a dominant set of managerial assumptions, which are the most characteristic of the managerial style a person has adopted, the concept of a backup set of assumptions is a useful one. An individual's backup theory is the one he uses when his dominant theory fails to get the desired results. It is the style he falls back on [Blake \& Mouton, 1964, p. 13]. 
There are at least two points of disparity, however, between the conceptualizations of Fiedler, and Blake and Mouton. One difference is that LPC is clearly a personality measure, while the Blake and Mouton formulation, although it does deal with attitudes, motivation, and managerial theories, is based primarily on observations of managerial behavior. The other difference is that while the Fiedler model implicitly places task orientation and interpersonal relations orientation as end points of a single continuum, Blake and Mouton and a preponderance of other researchers (e.g., Fleishman, Burtt, \& Harris, 1955; Kahn, 1958) maintain that these dimensions, at least in the behavioral domain, are very nearly independent. Irrespective of the degree of dependence or independence of these two dimensions, however, an LPC score may be viewed as a "forced choice" measure of the relative strength of an individual's motivational orientation toward interpersonal behavior as opposed to task behavior.

The present study examined data relevant to three specific predictions derived from Fiedler's most recent statement of the "contingency model," and further investigated the nature of LPC scores by separately examining leader task and interpersonal relations orientation scores, as well as combining them into an LPC equivalent, "forced preference" index. The predictions are as follows:

1. Correlations between measures of leaders' relative preference for interpersonal relations orientation, as opposed to a preference for task orientation, and work group effectiveness are negative in situations of low favorability, positive in situations of intermediate favorability, and more negative in situations of high favorability.

2. Correlations between measures of leaders' relative preference for interpersonal relations orientation and measures of their task-oriented behavior are increasingly positive in successive levels of increased situational favorability.

3. Correlations between measures of leaders' relative preference for interpersonal relations orientation and measures of their interpersonally oriented behavior are increasingly negative in successive levels of increased situational favorability.

\section{METHOD}

\section{Subjects}

The present sample consisted of 119 first-line supervisors from the production and maintenance departments of a metal casting and fabricating plant located near a small midwestern city. The groups they supervised ranged in size from three to 35 , and were engaged in a variety 
of relatively structured tasks involved in the operation and maintenance of production equipment, the movement and supply of materials, and the packing and shipping of the completed product.

\section{Measures}

Four separate types of measures were used in this study. They are personality-motivational measures of leader task and interpersonal relations orientation, observational measures of leader behavior, ratings of group effectiveness, and a measure of the quality of leader-member relations. The independent variables in this study were leader task and interpersonal orientation scores obtained from a subset of items of the Survey of Management Beliefs (SMB) questionnaire from the Institute for Social Research at the University of Michigan. The SMB questionnaire consisted of a series of statements to which the respondent marked a point on a nine-point scale ranging from "completely agree" to "completely disagree" for each statement. Supervisors' interpersonal relations orientation scores were derived from the subset of items of the SMB that make up an index called Human Relations. The six items in the index were as follows:

1. Management should rely more on mutual confidence and good relationships with people rather than on the exercise of authority to get things done.

2. The good manager must pay as much direct attention to keeping people working together as he does to seeing that the task gets done.

3. It is essential for the good manager to be sensitive to the feelings of others.

4. In business, emotions and feelings should be expressed and worked out.

5. Managers should be willing to try out new ways of doing things.

6. A manager should help others to express their own individuality.

Supervisors' task orientation scores were taken from a subset of items that very closely resemble the set of assumptions that McGregor (1960) labeled as "Theory X"; hence the index bore that name. The Theory X index contained the following items:

1. The most effective way to get people motivated and committed to a job is to instruct, direct, and use appropriate rewards and penalties.

2. Although a manager can be democratic with his employees, he must still structure their work for them.

3. A supervisor must keep a close check on his employees to see that they are doing a good job.

4. Employees prefer to be directed rather than making their own decision in their work.

5. In industry there must always be a unity of command. 
6. Being firm with employees is the best way to insure that they will do a good job.

7. A clear-cut hierarchy of authority and responsibility is essential in a business organization.

8. A manager must be a strong individualist.

A score on the Human Relations and Theory $\mathrm{X}$ indices was the sum of the question values divided by the number of questions in the index. A score representing relative dominance of a supervisor's task and interpersonal relations orientation was obtained by subtracting his Theory $\mathrm{X}$ score from his Human Relations score after the total set of scores on both the Human Relations and Theory X indices had been transformed into Z-score distributions. The scale score range, median, and internal consistency-reliability coefficient alpha for this "forced preference" for Human Relations orientation (FP-Human Relations), and for the Human Relations and Theory $\mathrm{X}$ indices, are shown in Table 1.

The remainder of the measures used in the study, six dependent variables and one control variable, were taken from subordinate responses to subsets of items of the Survey of Organizations (Taylor \& Bowers, 1970), a machine-scored, paper-and-pencil questionnaire developed at the Institute for Social Research at the University of Michigan. In all, five supervisory behavior measures were used. Four of the indices have been described elsewhere (Bowers \& Seashore, 1966). These indices and items incorporated in them were as follows.

Supervisory Support (Support)

42. How friendly and easy to approach is your supervisor?

44. When you talk with your supervisor, to what extent does he pay attention to what you're saying?

46. To what extent is your supervisor willing to listen to your problems?

Supervisory Goal Emphasis (Goal Emphasis)

48. How much does your supervisor encourage people to give their best effort?

TABLE 1

Minimum, Median, and Maximum Scores and Coefficient Alphas for Tast and Interpersonal Relations Orientation Mrasures

\begin{tabular}{lcccc}
\hline \multicolumn{1}{c}{ Measure } & Minimum & Median & Maximum & Coefficient alpha \\
\hline FP-human relations & 2.78 & 10.08 & 13.11 & .70 \\
Human relations & 1.25 & 7.67 & 9.00 & .72 \\
Theory X & 1.09 & 3.38 & 7.38 & .82 \\
\hline
\end{tabular}


50. To what extent does your supervisor maintain high standards of performance?

\section{Supervisory Work Facilitation (Work Facilitation)}

54. To what extent does your supervisor show you how to improve your performance?

56. To what extent does your supervisor provide the help you need so that you can schedule work ahead of time?

58. To what extent does your supervisor offer new ideas for solving job-related problems?

\section{Supervisory Interaction Facilitation (Interaction Facilitation)}

60. To what extent does your supervisor encourage the persons who work for him to work as a team?

62. To what extent does your supervisor encourage people who work for him to exchange opinions and ideas?

Supervisors' scores on the remaining supervisory behavior measure, Pressure for Production, were taken from subordinate responses to a single question from a supplement to the Survey of Organizations. This item was stated as follows:

On the job, to what extent do you feel pressure from your supervisor for better performance, over and above what you yourself think is reasonable?

The Work Group Effectiveness ratings were obtained from group members' responses to the following question from the Survey of Organizations:

On the basis of your experience and information, how would you rate your work group on effectiveness? How well does it do in fulfilling its mission or achieving its goals in comparison with other work groups in this organization?

1. The work group does a rather poor job.

2. Fair

3. Good

4. Very good

5. The work group does an excellent job.

The remaining measure, Leader-Member Relations, which was used as a control variable in this study, was derived from the following three items from the Survey of Organizations:

All in all, how satisfied are you with your supervisor? 
To what extent do you feel your supervisor has confidence and trust in you?

To what extent do you have confidence and trust in your supervisor?

The response alternatives on all of the questions from the Survey of Organizations used in this study were as follows:
1. To a very little extent
2. To a little extent
3. To some extent
4. To a great extent
5. To a very great extent

A supervisor's score for the multiple item indices was calculated by first finding a mean value across all of the items in the index for each subordinate in his group, and then computing a mean value across all the subordinates. This resulted in a set of scores with a potential range from one to five. The minimum, median, and maximum scale scores and the internal consistency-reliability coefficient alphas for the supervisory behavior, Work Group Effectiveness, and Leader-Member Relations measures are shown in Table 2.

\section{Procedure}

The Survey of Organizations questionnaire was completed by over $90 \%$ of the employees of the plant as part of an ongoing organizational research study, in groups of 50-200 with the assistance and supervision of a representative of the Institute for Social Research of the University of Michigan. The respondents were asked not to identify themselves on the questionnaire, and were assured of respondent anonymity. Each respondent was asked to identify his supervisor through the placement of a

TABLE 2

Minimum, Median, and Maximum Scores and Coeffictent Alphas for Supervisory Behavior, Work Group EFFECTIVEness, and

Leader-Member Relations Measures

\begin{tabular}{lcccc}
\hline \multicolumn{1}{c}{ Measure } & Minimum & Median & Maximum & $\begin{array}{c}\text { Coefficient } \\
\text { alpha }^{a}\end{array}$ \\
\hline Supervisory support & 1.00 & 3.28 & 4.67 & .91 \\
Supervisory goal emphasis & 1.00 & 3.31 & 5.00 & .80 \\
Supervisory work facilitation & 1.00 & 2.60 & 5.00 & .88 \\
Supervisory interact. Facilitation & 1.00 & 2.69 & 4.67 & .84 \\
Pressure for production & 1.50 & 3.40 & 5.00 & - \\
Work group effectiveness & 2.00 & 3.58 & 5.00 & - \\
Leader-member relations & 1.00 & 3.30 & 5.00 & .92 \\
\hline
\end{tabular}

a Not applicable to single item measures. 
designated supervisory identification number on his questionnaire. Work group scores were tabulated on the basis of these supervisory identification numbers. A few days later, each supervisor was given a copy of the Survey of Management Beliefs questionnaire by a representative of ISR, and was asked to complete it and mail it directly to the Institute. Completed questionnaires were received from 119 of 127 eligible supervisors.

\section{RESULTS}

The first step in the analysis of the data was the division of the sample into groups of high, medium, and low "situational favorability" on the basis of their scores on the Leader-Member Relations index. The remaining two components of "situational favorability" in the contingency model, task structure and leader position power, were assumed to be controlled through the selection of groups with similarly structured tasks and appointed leaders with approximately equal and relatively high position power. A separate analysis was carried out for the high, medium, and low groups. Pearson product-moment correlations were then computed between pairs of the three independent variables, FP-Human Relations, and Theory $\mathrm{X}$, and pairings of the independent variables with the six dependent variables (five supervisory behavior variables and Work Group Effectiveness). Possible cross contamination of the independent variable-dependent variable relationships due to intercorrelations of the Human Relations and Theory $\mathrm{X}$ indices was then eliminated by the computation of correlations between these measures and the six dependent variables, partialling out the effect of the other measure (Nunnally, 1967). The resulting correlations and the zero-order correlations between FP-Human Relations and the six dependent variables were then corrected for attenuation to achieve a more reliable estimate of the true independent-dependent variable relationships. (In the two instances where the dependent variable was a single item, 1.00 was substituted for a reliability estimate in the computational formula resulting in a more conservative estimate of the actual independent-dependent variable relationship.) The net effect of this procedure was a slight increase in the correlation levels which, in some cases, resulted in the latter values achieving statistical significance whereas the uncorrected values did not.

The significance of differences in independent-dependent variable correlations across groups was then computed using a technique described by Hays (1963, pp. 529-532). In those instances where a monotonic curve across groups was predicted, the significance of correlation differences between low and high situational favorability groups was determined. The predicted relationship for Work Group Effectiveness, however, was curvilinear, and in this case, the significance of correlation differences 
TABLE 3

Correlations between Independent Variable Parrs

\begin{tabular}{lc}
\hline \multicolumn{1}{c}{ Variable pair } & Correlation coefficient \\
\hline FP-human relations-human relations & .619 \\
FP-Human relations-theory X & -.619 \\
Human relations-theory X & .234 \\
\hline
\end{tabular}

from low to medium and from medium to high situational favorability were calculated.

The Pearson product-moment correlations among the independent variables for the total sample are shown in Table 3 . The high negative correlation between Human Relations and Theory X, predicted from the Fiedler model, was not found.

The zero-order correlations and correlations corrected for attenuation between FP-Human Relations and the six dependent variables for high, medium, and low situational favorability groups appear in Table 4. Of the 18 zero-order correlations and 18 correlations corrected for attenuation, five are significantly different from zero beyond the .05 level. Table 4 also contains the zero-order correlations, partial correlations, and correlations corrected for attenuation between Human Relations and Theory $\mathrm{X}$ scores and the six dependent variables for the high, medium, and low situational favorability groups. When the 36 correlations of each type were tested for significance, two of the zero-order, two of the partial, and seven of the corrected correlations exceeded the .05 level of significance. The corrected partial correlations between the three independent and six dependent variables are presented graphically in Figs. 1-6.

The differences and significance tests for Work Group Effectiveness correlations with the FP-Human Relations index, from low to medium $(.48, p<.02)$, and from medium to high groups $(-.46, p<.02)$, are shown in Table 5 . Both of these differences were in the predicted direction.

Table 5 also contains the differences and significance tests for Work Group Effectiveness correlations with the Human Relations and Theory $\mathrm{X}$ indices, from low to medium and from medium to high groups. Of the four differences for these variables, two were significant beyond the .05 level.

Differences were obtained between low and high situational favorability groups for the correlations of the supervisory behavior indices with FPHuman Relations, Human Relations, and Theory X indices. Table 6 presents these differences and their significance levels. The correlation differences for the two interpersonal relations-oriented supervisory behavior indices, Support $(-.51, p<.02)$ and Interaction Facilitation 


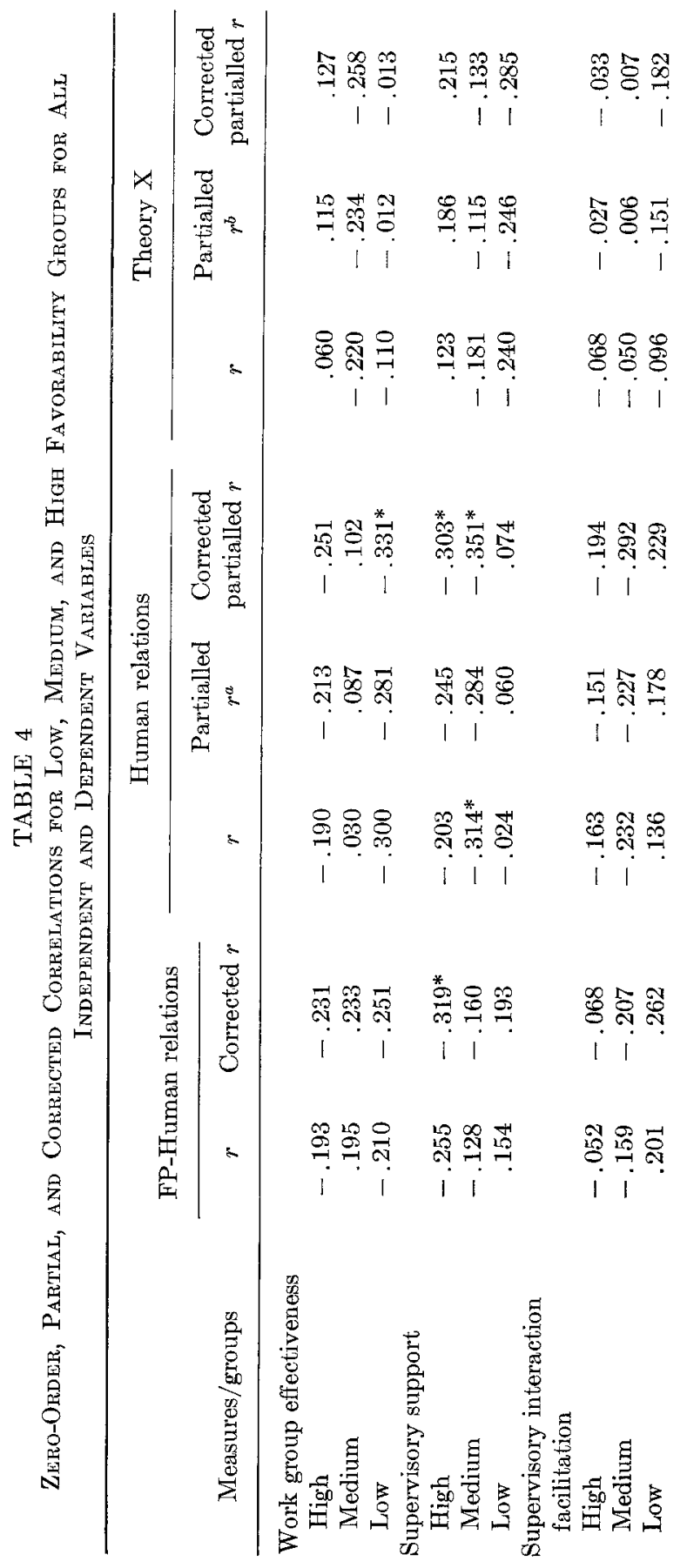




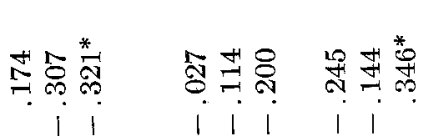

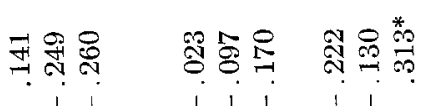

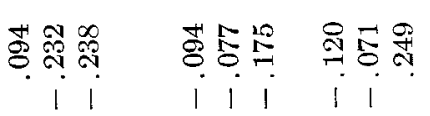

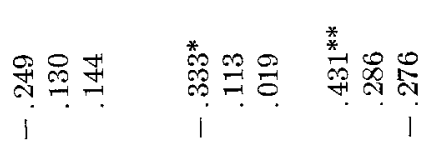

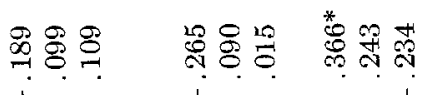

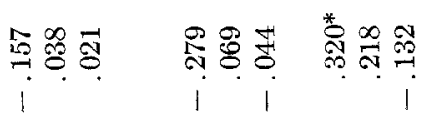

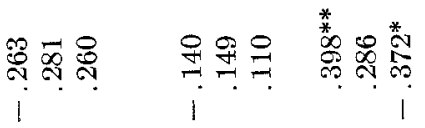



$\dot{\overrightarrow{5}}$

突要

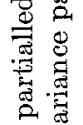

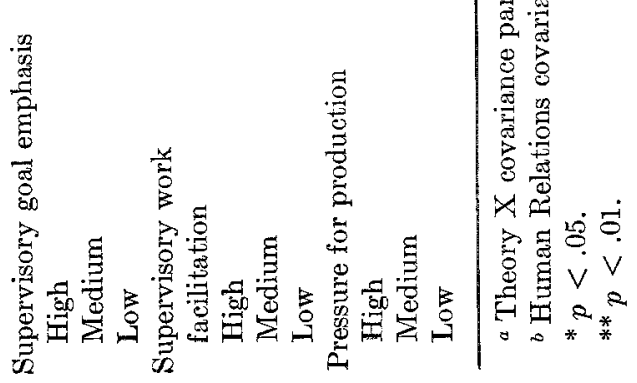




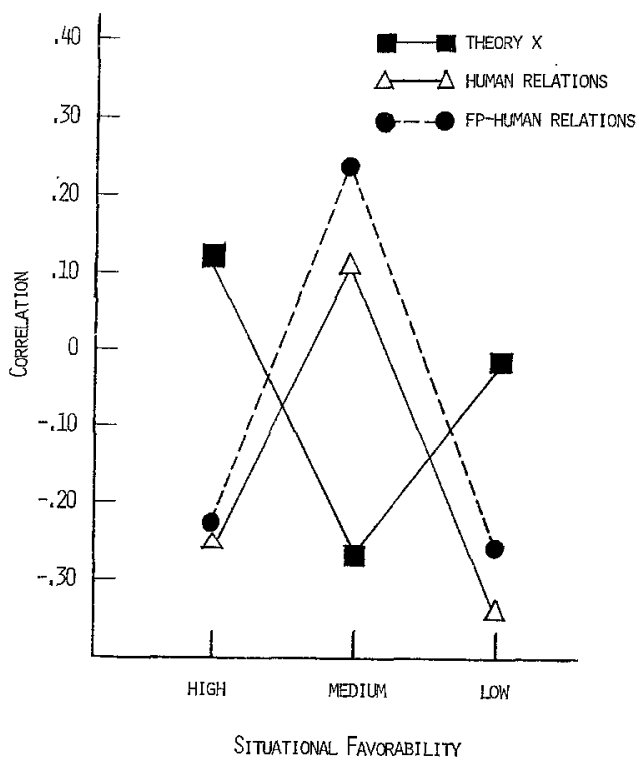

FIG. 1. Correlations of Work Group Effectiveness with Theory X, Human Relations, and FP-Human Relations for Varying Levels of Situational Favorability.

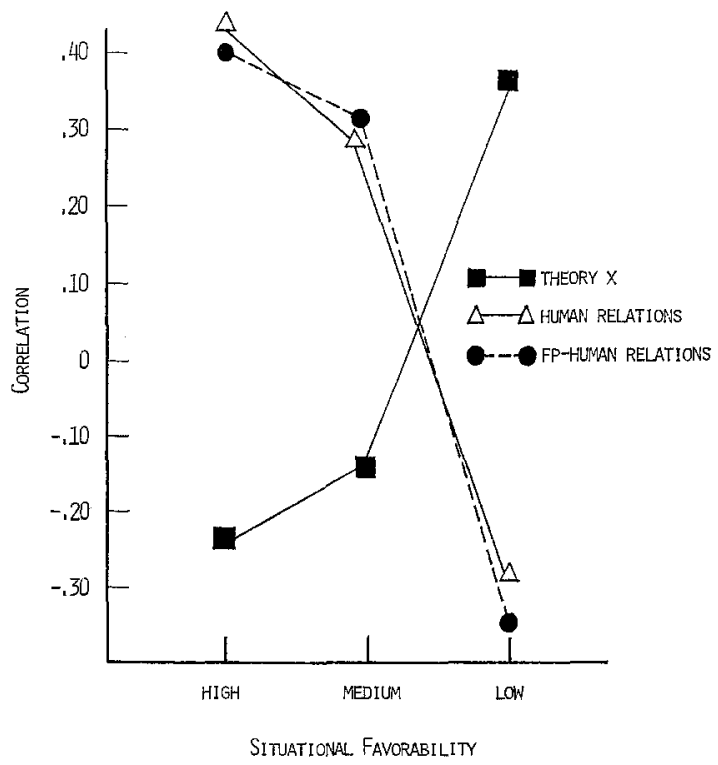

Fra. 2. Correlations of Pressure for Production with Theory X, Human Relations, and FP-Human Relations for Varying Levels of Situational Favorability. 


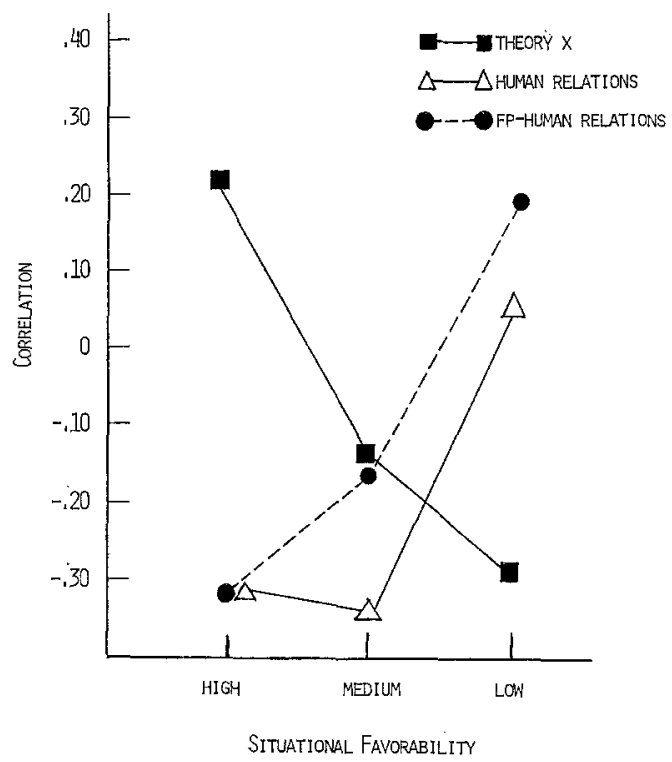

Fia. 3. Correlations of Support with Theory X, Human Relations, and FP-Human Relations for Varying Levels of Situational Favorability.

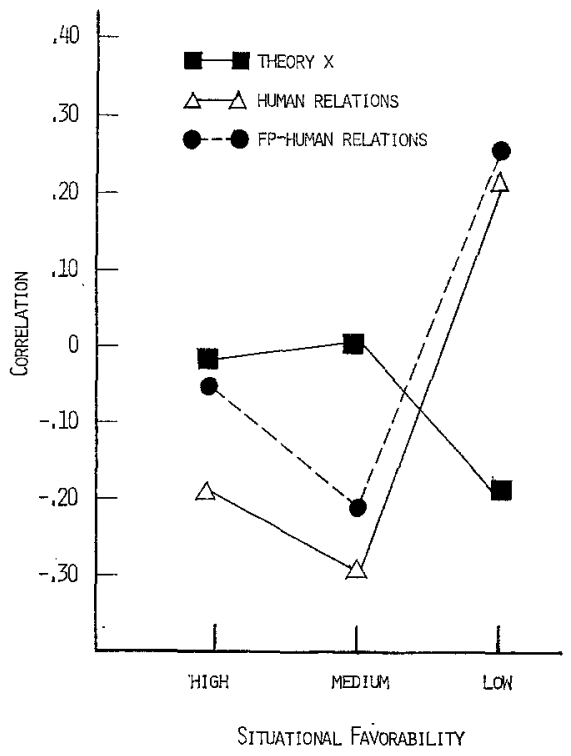

FIG. 4. Correlations of Interaction Facilitation with Theory X, Human Relations, and FP-Human Relations for Varying Levels of Situational Favorability. 


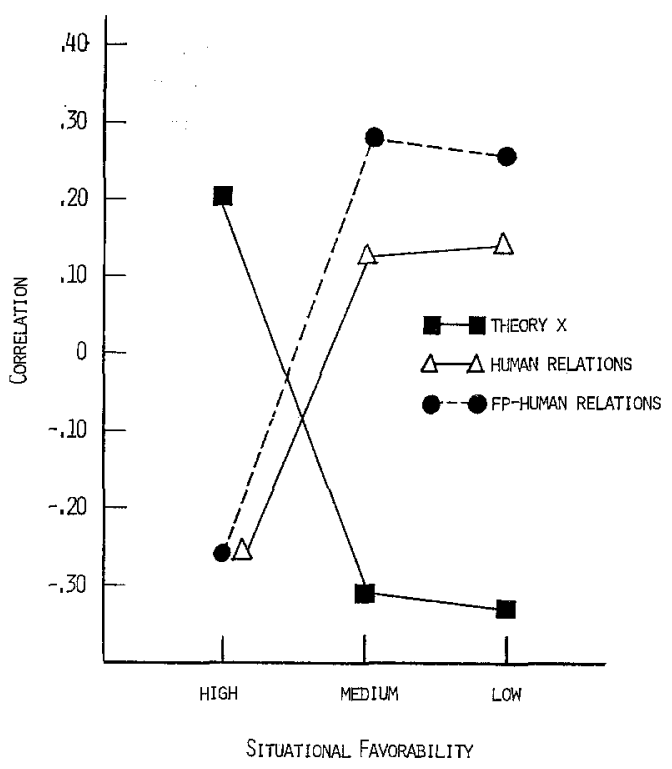

FIG. 5. Correlations of Goal Emphasis with Theory X, Human Relations, and FP-Human Relations for Varying Levels of Situational Favorability.

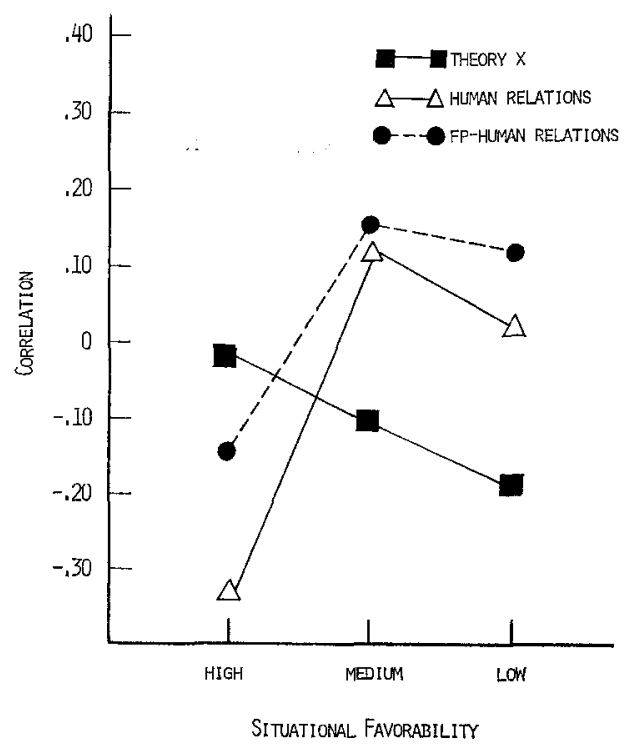

FIG. 6. Correlations of Work Facilitation with Theory $\mathrm{X}$, Human Relations, and FP-Human Relations for Varying Levels of Situational Favorability. 
TABLE 5

Cross-Level Differences of Correlations between Measures of Work Grodp Effectiveness, and Leader Orientation, and

Their Significance Levels

\begin{tabular}{lccccc}
\hline & \multicolumn{4}{c}{ Situational favorability } \\
\cline { 2 - 3 } & \multicolumn{2}{c}{ High minus medium } & & Medium minus low \\
\cline { 2 - 3 } \cline { 5 - 6 } Leader orientation & $\begin{array}{c}\text { Correlation } \\
\text { difference }\end{array}$ & $\begin{array}{c}\text { Significance } \\
\text { level }\end{array}$ & & $\begin{array}{c}\text { Correlation } \\
\text { difference }\end{array}$ & $\begin{array}{c}\text { Significance } \\
\text { level }\end{array}$ \\
\hline FP-human relations & -.460 & .02 & & .480 & .02 \\
Human relations & -.353 & .07 & & .433 & .03 \\
Theory X & .385 & .05 & & -.245 & .14 \\
\hline
\end{tabular}

$(-.33, p<.07)$ were in the predicted direction (see Figs. 3 and 4$)$. The results for the task-oriented supervisory behavior indices were much less orderly. While the correlation difference for Pressure for Production $(.77, p<.01)$ was statistically significant in the predicted direction (see Fig. 2), the correlation of differences for Goal Emphasis $(-.52, p<.01)$ and Work Facilitation $(-.25, p<.14)$ were opposite the predicted direction. Of the 10 correlation differences between the supervisory behavior indices and the Human Relations and Theory $\mathrm{X}$ indices, seven were significant beyond the .05 level (see Figs. 5 and 6 ).

\section{DISCUSSION}

The results of this study generally support the basic notion of the contingency model--that the situation moderates the relationship between attributes and behavior of leaders and the effectiveness of their groups performance. These findings also represent an extension of the model due to the fact that an entire set of conceptually similar but operationally different independent, dependent, and control variables was used.

\section{Leader Orientation and Work Group Effectiveness}

The results confirmed the prediction that groups supervised by interpersonal relations-oriented leaders (high FP-Human Relations) would perform more effectively in situations of intermediate favorability than they would in situations of either high or low favorability (see Fig. 1). Although these findings are consistent with those reported by Fiedler (1971b), they should be interpreted with caution because, although the predicted correlation differences are highly significant, the absolute value of the correlation coefficients between FP-Human Relations and Work Group Effectiveness were not statistically significant from zero in any of the situational favorability groups. 


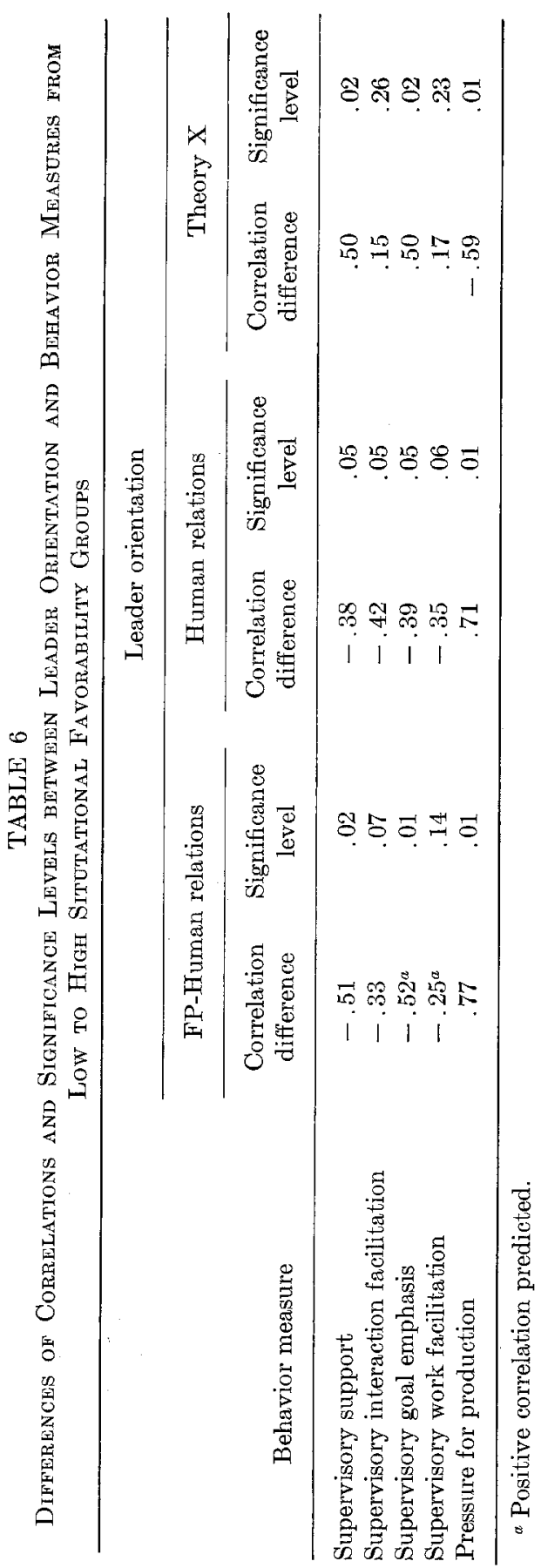




\section{Leader Orientation and Leader Behavior}

The results verify the existence, as predicted, of a positive correlation between leaders' interpersonal relations orientation scores (FP-Human Relations) and the two interpersonally oriented behavior measures (Support and Interaction Facilitation) in the low favorability situation, and of a significantly different negative correlation between these same variables in the high favorability situation (see Figs. 3 and 4). These findings support Fiedler's $(1970,1971 a)$ assertion that leaders will be concerned with the achievement of goals consistent with their primary orientation (e.g., high LPC, satisfying relationships) in stressful situations, while under more favorable circumstances, leaders will focus most of their attention on the achievement of their lower order goals (e.g., high LPC, task-related goals). In addition, the correlations between FP-Human Relations and Pressure for Production (see Fig. 2) in the low and high favorability situations further substantiate the Fiedler analysis in that they are an empirical verification of the remaining half of the model. Exactly the opposite relationships were found, however, for the remaining two task-related behavior measures, Goal Emphasis and Work Facilitation (see Figs. 5 and 6). One explanation for these apparently discrepant findings is that these two task measures contain elements of interpersonally oriented behavior. This possibility is very strongly suggested by the fact that Goal Emphasis and Work Facilitation have high positive correlations with Support and Interaction Facilitation (all above .80) and low negative correlations $(-.20$ and -.25$)$ with Pressure for Production. In addition, a review of the questions from which the Goal Emphasis and Work Facilitation indices were taken suggests that these indices measure behavior with both interpersonal and task elements, such as the degree to which supervisors provide help in learning, scheduling, and performing the job, have high standards, and encourage individuals to give their best effort.

\section{FP-Human Relations, Human Relations, and Theory X}

The occurrence of a low positive correlation (.23) in this study between the Human Relations index and the Theory $\mathrm{X}$ index calls into question one of the implicit assumptions of the Fiedler model, namely, that task orientation and interpersonal relations orientation are the opposite extremes of a single continuum. The current findings as well as those of Kahn (1956), Fleishman et al. (1955), and Blake and Mouton (1964) support the contradictory conclusion that task orientation and interpersonal relations orientation are actually independent dimensions.

Fiedler asserted $(1970$, p. 5$)$ that individuals may be classified as being 
either task- or human relations-oriented on the basis of a single score. The fact that these dimensions appear to be independent raises the possibility, however, that this practice introduces contamination into the resultant findings. This is particularly true of LPC scores since they are presumably a function of two underlying distributions, neither of which is directly measured. The evidence from this study, however, does not substantiate this potential criticism of the Fiedler methodology. A comparison of the dependent variable correlations with the FP-Human Relations, Human Relations, and Theory X indices (see Figs. 1-6), on the contrary, reveals that substantially the same conclusions would be drawn using any one of the three as a measure of supervisors' primary orientation.

\section{CONCLUSIONS}

Data collected using the Survey of Organizations and Survey of Management Beliefs questionnaires from members and supervisors of 119 work groups in the maintenance and production departments of a metal fabricating plant were analyzed in terms of four specific predictions based on the "contingency model" proposed by Fiedler (1964, 1970). Leader "forced preference" interpersonal relations orientation scores were correlated with leader behavior measures and Work Group Effectiveness scores in three levels of situational favorability. The results generally supported the contingency model with some modifications. Specifically the data suggested the following conclusions about the relationship between supervisors' primary orientation and the way they actually supervise:

1. In a low favorability situation, interpersonal relations-oriented supervisors demonstrated concern and support for subordinates and exerted very little pressure on them for production.

2. In a low favorability situation, task-oriented supervisors exerted pressure on subordinates for production and demonstrated relatively little concern and support for them.

3. In a high favorability situation, interpersonal relations-oriented supervisors demonstrated relatively less concern and support for subordinates and put more pressure on them for production.

4. In a high favorability situation, task-oriented supervisors exerted less pressure on subordinates for production and demonstrated more concern and support for them.

Stated more generally, in very unfavorable situations, supervisors directed most of their behavior toward achievement of their primary goals, while in very favorable situations, they concentrated less on the achievement of primary goals and more on the achievement of secondary 
goals. The results suggested that if you ask a supervisor how leaders should behave, he would accurately describe to you the way he would behave in an unfavorable situation, but his description would be increasingly less accurate as the situation became more favorable.

The data also suggested the following conclusions about the relationship between supervisors' primary orientation and the effectiveness of the groups they supervise:

1. Groups supervised by task-oriented supervisors were more effective in situations of either high or low favorability than in situations of intermediate favorability.

2. Groups supervised by interpersonal relations-oriented supervisors were more effective in situations of intermediate favorability than in situations of either high or low favorability.

\section{REFERENCES}

Blake, R. R., \& Mouton, J. S. The managerial grid. Houston, TX: Gulf Publishing Co., 1964.

Bowers, D., \& SeAshore, S. Predicting organizational effectiveness with a four-factor theory of leadership. Administrative Science Quarterly, 1966, 11, 238-263.

FiedLer, F. E. A contingency model of leadership effectiveness. In L. Berkowitz (Ed.), Advances in experimental social psychology. New York: Academic Press. 1964.

Finder, F. E. A theory of leadership effectiveness. New York: McGraw-Hill, 1967.

FiedLer, F. E. Personality and situational determinants of leadership effectiveness. In D. Cartwright \& A. Zander (Eds.), Group dynamics: Research and theory. (3rd ed.) Evanston, IL: Row, Peterson and Co., 1968.

FiedLer, F. E. Personality motivational systems, and behavior of high and low LPC persons. Technical Report, Office of Naval Research, 1970.

Fieduer, F. E. Leadership. New York: General Learning Corp., 1971 (a).

Fiedler, F. E. Validation and extension of the contingency model of leadership effectiveness. Psychological Bulletin, 1971, 76, 128-148 (b).

Fleishman, E. A., Harris, E. F., \& Burtt, H. E. Leadership and supervision in industry. Columbus, $\mathrm{OH}$ : Personnel Research Board, Ohio State University, 1955.

Graen, G., Alvares, K., Orris, J. B., \& Marletta, J. A. Contingency model of leadership effectiveness: Antecedent and evidential results. Psychological Bulletin, 1970, 74, 285-296.

Hays, W. L. Statistics for psychologists. New York: Holt, Rinehart \& Winston, 1963.

KaHN, R. L. The prediction of productivity. Journal of Social Issues, 1956, 12, 41-49.

Maslow, A. H. The authoritarian character structure. Journal of Social Psychology, $1943,18,241-270$.

MaGReGor, D. The human side of enterprise. New York: McGraw-Hill, 1960.

Nunnaliy, J. C. Psychometric theory. New York: McGraw-Hill, 1967.

TAYlor, J., \& Bowers, D. The survey of organizations: Toward a machine-scored, standardized questionnaire instrument. Technical Report, Office of Naval Research, 1970.

RECEIVED: March 10, 1972 